VoL. 50 (1994) [73-80]

\title{
SOME COINCIDENCE THEOREMS AND APPLICATIONS
}

\author{
Xie Ping Ding and E. Tarafdar
}

In this paper, we establish a new coincidence theorem for a Browder type set-valued mapping and an upper semi-continuous set-valued mapping with compact acyclic values in an $H$-space which generalises some recent results in the literature. As applications we obtain two $\mathrm{Ha}$ type coincidence theorems and existence theorems of maximal elements for preference correspondences.

\section{INTRODUCTION}

Let $X$ and $Y$ be two nonempty sets and $S: X \rightarrow 2^{Y}, T: Y \rightarrow 2^{X}$ be two setvalued mappings, where $2^{A}$ denotes the family of all subsets of the set $A$. Following Browder [4], a point $\left(x_{0}, y_{0}\right) \in X \times Y$ is said to be a coincident point if $y_{0} \in S\left(x_{0}\right)$ and $x_{0} \in T\left(y_{0}\right)$. There are other equivalent definitions for a coincidence point of two mappings, see for example, Park [15] and Ding [5]. Coincidence theory is a generalisation of fixed point theory which has become an important and fundamental tool in treating some nonlinear problems.

In the present paper, we establish a new coincidence theorem for a Browder type set-valued mapping and an upper semi-continuous set-valued mapping with compact acyclic values in an $H$-space which generalise some recent results of Komiya [13], Sessa [16], Mehta and Sessa [14] and Tarafdar [21]. As applications, we obtain some existence theorems of maximal elements for preference correspondences.

\section{Preliminaries}

Let $X$ and $Y$ be topological spaces, $\mathcal{F}(Y)$ the family of all nonempty finite subsets of $Y$ and $F: X \rightarrow 2^{Y}$ a set-valued mapping (or correspondence). For $A \subset X, B \subset Y$ and $y \in Y$, let

$$
\begin{aligned}
F(A) & =\bigcup_{x \in A} F(x), \quad F^{+}(B)=\{x \in X: \emptyset \neq F(x) \subset B\} \quad \text { and } \\
F^{-1}(y) & =\{x \in X: y \in F(x)\}
\end{aligned}
$$

Received 30th Setpember, 1993

The work by the first author was supported by the National Natural Science Foundation of China and by the Ethel Raybould Fellow scholarship of the University of Queensland, Australia.

Copyright Clearance Centre, Inc. Serial-fee code: 0004-9729/94 \$A2.00+0.00. 
$F$ is said to be upper semi-continuous (in short, u.s.c.) on $X$ if for each open subset $V$ of $Y, F^{+}(V)$ is open in $X$. A subset $D$ of $X$ is said to be compactly open (respectively compactly closed) if $D \cap K$ is open (respectively closed) in $K$ for each nonempty compact subset $K$ of $X$.

The following notions were introduced by Bardaro and Cepptelli [2]. A pair $\left(Y,\left\{\Gamma_{A}\right\}\right)$ is called an $H$-space if $Y$ is a topological space and $\left\{\Gamma_{A}\right\}$ a family of contractible subsets of $Y$ indexed by $A \in \mathcal{F}(Y)$ such that $\Gamma_{A} \subset \Gamma_{A^{\prime}}$ whenever $A \subset A^{\prime}$. A nonempty subset $D$ of an $H$-space $\left(Y,\left\{\Gamma_{A}\right\}\right)$ is said to be

(i) $H$-convex if $\Gamma_{A} \subset D$ for each $A \in \mathcal{F}(D)$,

(ii) weakly $H$-convex if $\Gamma_{A} \cap D$ is contractible for each $A \in \mathcal{F}(D)$,

(iii) $H$-compact in $Y$ if for each $A \in \mathcal{F}(Y)$, there exists a compact, weakly $B$-convex subset $D_{A}$ of $Y$ such that $D \cup A \subset D_{A}$.

Recall that a nonempty topological space is an acyclic space if all of its reduced Cech homology groups over the rationals vanish. In particular, any contractible space is acyclic, and hence any convex or star-shaped set in a topological vector space is acyclic. For a topological space $X$, we shall denote by $k a(X)$ the family of all compact acyclic subsets of $X$.

Let $\Delta_{n}$ be the standard $n$-dimensional simplex with vertices $e_{0}, e_{1}, \ldots, e_{n}$. If $J$ is a nonempty subset of $\{0,1, \ldots, n\}, \Delta_{J}$ will denote the convex hull of the vertices $\left\{e_{j}: j \in J\right\}$.

The following Lemma, the proof of which is contained in the proof of Theorem 1 of Horvath [12], will be the basic tool for our purpose. (See also, Ding and Tan [6].)

LEMMA 2.1. Let $Y$ be a topological space. For each nonempty subset $J$ of $\{0,1, \ldots, n\}$, let $\Gamma_{J}$ be a contractible subset of $Y$. If $J \subset J^{\prime}$ implies $\Gamma_{J} \subset \Gamma_{J^{\prime}}$ then there exists a continuous mapping $f: \Delta_{n} \rightarrow Y$ such that $f\left(\Delta_{J}\right) \subset \Gamma_{J}$ for each nonempty subset $J$ of $\{0,1, \ldots, n\}$.

The following result is Lemma 1 of Shioji in [17].

LEMMA 2.2. Let $\Delta_{n}$ be an $n$-dimensional simplex with the Euclidean topology and $W$ be a compact topological space. Let $\psi: W \rightarrow \Delta_{n}$ be a single-valued continuous mapping and $T: \Delta_{n} \rightarrow k a(W)$ be u.s.c. Then there exists a point $x^{*} \in \Delta_{n}$ such that $x^{*} \in \psi\left(T\left(x^{*}\right)\right)$.

\section{Coincidence Theorems}

In this section, we shall establish some new coincidence theorems which generalise some recent results in the literature.

THEOREM 3.1. Let $K$ be a nonempty compact subset of a topological space $X$ and $\left(Y,\left\{\Gamma_{A}\right\}\right)$ an $H$-space. Let $G: X \rightarrow 2^{Y}$ and $T: Y \rightarrow k a(K)$ be set-valued 
mappings such that

(i) $T$ is u.s.c. on $Y$,

(ii) for each $x \in X, G(x)$ is $H$-convex and for each $y \in Y, G^{-1}(y)$ contains a compactly open subset $O_{y}$ of $X\left(O_{y}\right.$ may be empty for some $\left.y\right)$ such that $K \subset \bigcup_{y \in Y} O_{y}$.

Then there exist $x_{0} \in K$ and $y_{0} \in Y$ such that $x_{0} \in T\left(y_{0}\right)$ and $y_{0} \in G\left(x_{0}\right)$.

Proof: Since $O_{y}$ is compactly open for each $y \in Y$ and $K$ is compact, by (ii), there exists a finite subset $\left\{y_{0}, y_{1}, \ldots, y_{n}\right\}$ of $Y$ such that $K=\bigcup_{i=0}^{n}\left(O_{y_{i}} \cap K\right)$. For each nonempty subset $J$ of $\{0,1, \ldots, n\}$, let $F_{J}=F_{\left\{y_{j}\right\}_{j \in J}}$. Then clearly $F_{J} \subset F_{J^{\prime}}$ whenever $J \subset J^{\prime}$. By Lemma 2.1, there is a continuous mapping $g: \Delta_{n} \rightarrow Y$ such that $g\left(\Delta_{J}\right) \subset F_{J}$ for each nonempty subset $J$ of $\{0,1, \ldots, n\}$. By (i), $T: Y \rightarrow k a(K)$ is u.s.c. and hence the composition mapping $T \circ g: \Delta_{n} \rightarrow k a(K)$ is also u.s.c. Now let $\left\{f_{0}, f_{1}, \ldots, f_{n}\right\}$ be a partition of unity subordinate to the open covering $\left\{O_{y_{i}} \cap K\right\}_{i=0}^{n}$. Define a mapping $f: K \rightarrow \Delta_{n}$ by

$$
f(x)=\sum_{i=0}^{n} f_{i}(x) e_{i} \quad \text { for each } \quad x \in K .
$$

Clearly, $f$ is continuous. By Lemma 2.2, there exists a point $x^{*} \in \Delta_{n}$ such that $x^{*} \in f\left(T \circ g\left(x^{*}\right)\right)$ so that there exists a point $x_{0} \in T \circ g\left(x^{*}\right) \subset K$ such that $x^{*}=$ $f\left(x_{0}\right)=\sum_{i=0}^{n} f_{i}\left(x_{0}\right) e_{i}$. Let $J\left(x_{0}\right)=\left\{i \in\{0,1, \ldots, n\}: f_{i}\left(x_{0}\right) \neq 0\right\}$, then

$$
x^{*}=\sum_{i \in J\left(x_{0}\right)} f_{i}\left(x_{0}\right) e_{i} \in \Delta_{J\left(x_{0}\right)}
$$

and for each $i \in J\left(x_{0}\right), x_{0} \in O_{y_{i}} \cap K \subset O_{y_{i}} \subset G^{-1}\left(y_{i}\right)$. It follows that $y_{i} \in G\left(x_{0}\right)$ for each $i \in J\left(x_{0}\right)$. Since $G\left(x_{0}\right)$ is $H$-convex, we have

$$
g\left(x^{*}\right) \in g\left(\Delta_{J\left(x_{0}\right)}\right) \subset F_{J\left(x_{0}\right)} \subset G\left(x_{0}\right)
$$

Let $y_{0}=g\left(x^{*}\right)$. Then we have $x_{0} \in T\left(y_{0}\right)$ and $y_{0} \in G\left(x_{0}\right)$.

Remark 3.1. Theorem 3.1 improves and generalises Theorem 2.3 of Mehta and Sessa [14] in several aspects and hence in turn, generalises Theorem 7 of Sessa [16], Theorem 1 of Komiya [13], Theorem 1 of Browder [4] and Theorem 1 of Tarafdar [18].

COROLlary 3.1. Let $K$ be a nonempty compact subset of a topological space $X$ and $\left(Y,\left\{\Gamma_{A}\right\}\right)$ an $H$-space. Let $G: X \rightarrow 2^{Y}$ be a set-valued mapping such that for each $x \in X, G(x)$ is $H$-convex and for each $y \in Y, G^{-1}(y)$ contains a compactly open 
subset $O_{y}$ of $X\left(O_{y}\right.$ may be empty for some $y$ ) such that $K \subset \bigcup_{y \in Y} O_{y}$. Then for any continuous mapping $t: Y \rightarrow K$, there exists a point $y_{0} \in Y$ such that $y_{0} \in G\left(t\left(y_{0}\right)\right)$.

Proof: Define a mapping $T: Y \rightarrow 2^{K}$ by

$$
T(y)=\{t(y)\} \quad \text { for each } \quad y \in Y .
$$

Then $T: Y \rightarrow k a(K)$ is u.s.c. By Theorem 3.1, there exist $x_{0} \in K$ and $y_{0} \in Y$ such that $x_{0} \in T\left(y_{0}\right)=\left\{t\left(y_{0}\right)\right\}$ and $y_{0} \in G\left(x_{0}\right)$. Hence we must have $y_{0} \in G\left(t\left(y_{0}\right)\right)$.

REMARK 3.2. Corollary 3.1 is a version of Corollary 2.2 of Tarafdar [21].

Theorem 3.2. Let $X$ be a Hausdorff locally convex topological vector space, $\left(Y,\left\{\Gamma_{A}\right\}\right)$ be a compact $H$-space, $T: Y \rightarrow 2^{X}$ be u.s.c. with closed values and $g: Y \rightarrow$ $X$ be continuous such that

(i) for each $y \in Y, T(y) \cap g(Y)$ is a non-empty acyclic space,

(ii) for each $x \in g(Y), \lambda>0$ and any continuous semi-norm $p$ on $X$, the set $\{y \in Y: p(g(y)-x)<\lambda\}$ is $H$-convex.

Then there exists $y_{0} \in Y$ such that $g\left(y_{0}\right) \in T\left(y_{0}\right)$.

Proof: Assume that the conclusion is not true. Then $g(y) \notin T(y)$ for all $y \in Y$. By using an argument similar to the one in the proof of Theorem 2 of $\mathrm{Ha}$ [11], there exist $\lambda>0$ and a continuous semi-norm $p$ on $X$ such that

$$
p(g(y)-x)>\lambda \text { for all } y \in Y \quad \text { and } \quad x \in T(y) .
$$

Clearly $g(Y)$ is a compact subset of $X$. Define a mapping $T^{*}: Y \rightarrow 2^{g(Y)}$ by

$$
T^{*}(y)=T(y) \cap g(Y)
$$

By Theorem 3.1.8 of Aubin and Ekeland [1] and the condition (i), $T^{*}: Y \rightarrow k a(g(Y))$ is u.s.c. Define $G: g(Y) \rightarrow 2^{Y}$ by

$$
G(x)=\{y \in Y: p(g(y)-x)<\lambda\}, \quad \forall x \in g(Y) .
$$

By the condition (ii), for each $x \in g(Y), G(x)$ is $H$-convex. It follows from the continuity of $p$ that for each $y \in Y, G^{-1}(y)=\{x \in g(Y): p(g(y)-x)<\lambda\}$ is an open subset of $g(Y)$. For each $x \in g(Y)$, there is some $y_{1} \in Y$ such that $x=g\left(y_{1}\right)$ and hence $y_{1} \in G(x)$ and $x \in G^{-1}\left(y_{1}\right)$. Hence $g(Y)=\bigcup_{y \in Y} G^{-1}(y)$. By Theorem 3.1 , there exist $x_{0} \in g(Y)$ and $y_{0} \in Y$ such that $x_{0} \in T^{*}\left(y_{0}\right)=T\left(y_{0}\right) \cap g(Y)$ and $y_{0} \in G\left(x_{0}\right)$. Hence, we have $p\left(g\left(y_{0}\right)-x_{0}\right)<\lambda$ and $x_{0} \in T\left(y_{0}\right)$ which contradicts (3.1). Therefore there exists a point $y_{0} \in Y$ such that $g\left(y_{0}\right) \in T\left(y_{0}\right)$. 
Remark 3.3. Theorem 3.2 generalises Theorem 2.2 of Mehta and Sessa [14] in several aspects.

Theorem 3.3. Let $X$ be a Hausdorf locally convex topological vector space, $\left(Y,\left\{\Gamma_{A}\right\}\right)$ be a compact $H$-space. $T: Y \rightarrow 2^{X}$ be u.s.c. with closed values and $g: Y \rightarrow X$ be continuous such that

(i) for each $y \in Y, g^{-1}(T(y))$ is a non-empty acyclic set.

(ii) for each closed convex subset $C$ of $X, g^{-1}(C)$ is an $H$-convex subset of $Y$.

Then there exists a point $y_{0} \in Y$ such that $g\left(y_{0}\right) \in T\left(y_{0}\right)$.

Proof: Assume that the conclusion does not hold, then, by an argument similar to that in the proof of Theorem 3.2, there exist $\lambda>0$ and a continuous semi-norm $p$ on $X$ such that

$$
p(g(y)-x)>\lambda, \text { for all } y \in Y \text { and } x \in T(y) .
$$

Define mappings $T^{*}: G=Y \rightarrow 2^{Y}$ by

$$
\begin{aligned}
T^{*}(y) & =g^{-1}(T(y)) \text { for each } y \in Y, \\
G(y) & =\{z \in Y: p(g(z)-g(y))<\lambda\} \text { for each } y \in Y .
\end{aligned}
$$

Since $g$ is continuous and $T$ is u.s.c., it is easy to see that $T^{*}$ has a closed graph. Note that $Y$ is compact, so by Corollary 3.1.9 of Aubin and Ekeland [1], $T^{*}$ is u.s.c. with compact values. By (i), $T^{*}: Y \rightarrow k a(X)$ is u.s.c. For each $y \in Y$ and $A=$ $\left\{y_{1}, \ldots, y_{n}\right\} \subset G(y)$, let $u_{i}=g\left(y_{i}\right), i=1, \ldots, n$ and hence $y_{i} \in g^{-1} u_{i}, i=1, \ldots, n$ and $A \subset g^{-1}\left[\operatorname{co}\left(u_{1}, \ldots, u_{n}\right)\right]$. By (ii), $g^{-1}\left[\operatorname{co}\left(u_{1}, \ldots, u_{n}\right)\right]$ is $H$-convex and so $\Gamma_{A} \subset$ $g^{-1}\left[\operatorname{co}\left(u, \ldots, u_{n}\right)\right]$. For any $z \in \Gamma_{A}$, there exist $\lambda_{i} \geqslant 0, i=1, \ldots, n$ with $\sum_{i=1}^{n} \lambda_{i}=1$ such that $g(z)=\sum_{i=1}^{n} \lambda_{i} u_{i}$. Note that $y_{i} \in G(y)$ for $i=1, \ldots, n$. It follows that

$$
\begin{aligned}
p(g(z)-g(y)) & =p\left(\sum_{i=1}^{n} \lambda_{i} u_{i}-g(y)\right)=p\left(\sum_{i=1}^{n} \lambda_{i}\left(g\left(y_{i}\right)-g(y)\right)\right) \\
& \leqslant \sum_{i=1}^{n} \lambda_{i} p\left(g\left(y_{i}\right)-g(y)\right)<\lambda
\end{aligned}
$$

and hence $\Gamma_{A} \subset G(y)$ and $G(y)$ is $H$-convex. By the continuity of $p$ and $g$, for each $z \in Y$,

$$
G^{-1}(z)=\{y \in Y: p(g(z)-g(y))<\lambda\}
$$

is open in $Y$. For each $y \in Y$, we have $y \in G(y)$ and hence $Y=\bigcup_{y \in Y} G(y)$. By Theorem 3.1 there exist $z_{0}, y_{0} \in Y$ such that $y_{0} \in T^{*}\left(z_{0}\right)=g^{-1}\left(T\left(z_{0}\right)\right)$ and $z_{0} \in G\left(y_{0}\right)$. Hence we have $g\left(y_{0}\right) \in T\left(z_{0}\right)$ and $p\left(g\left(z_{0}\right)-g\left(y_{0}\right)\right)<\lambda$ which contradicts (3.2). Hence there exists a point $y_{0} \in Y$ such that $g\left(y_{0}\right) \in T\left(y_{0}\right)$. 
REMARK 3.4. Theorem 3.3 improves Theorem 2 of Ha [11], Theorem 1 of Fan [9] and Theorem 2 of Fan [10].

\section{Existence of Maximal Elements}

Let $X$ and $Y$ be two topological spaces and $T: X \rightarrow 2^{Y}$ be a preference correspondence. A point $x \in X$ is said to be a maximal element of the preference correspondence $T$ if $T(x)=\emptyset$. The existence theorems of maximal elements have become an important tool in proving the equilibrium existence of abstract economics or generalised games, see for example, Borglin and Keiding [3], Yannelis and Prabhakar [22], Ding and Tan $[\mathbf{7}, \mathbf{8}]$ and Tarafdar $[20]$.

THEOREM 4.1. Let $K$ be a nonempty compact subset of a topological space $X$ and $\left(Y,\left\{\Gamma_{A}\right\}\right)$ an $H$-space. Let $G: X \rightarrow 2^{Y}$ and $T: Y \rightarrow 2^{K}$ be two correspondences. Suppose that

(i) $T$ is u.s.c. such that for each $y \in Y, T(y)$ is either an empty set or a closed acyclic set,

(ii) for each $y \in Y$, some $Q^{-1}(y)$ contains a compactly open subset $O_{y}$ of $X$ ( $O_{y}$ may be empty for some $y$ ) such that $K \subset \bigcup_{y \in Y} O_{y}$ where $Q(x)=H-c o(G(x))$, the $H$-convex hull of $G(x)$, (see Tarafdar [19]), for each $x \in X$,

(iii) for each $(x, y) \in K \times Y, x \in T(y)$ implies $y \notin Q(x)$.

Then either $T$ has a maximal element in $Y$ or $Q$ has a maximal element in $K$.

Proof: Assume that both $T$ and $Q$ do not have maximal elements. Then, by (i), $T: Y \rightarrow k a(K)$ is u.s.c. By (ii), for each $x \in X, Q(x)$ is $H$-convex and for each $y \in Y, Q^{-1}(y)$ contains a compactly open subset $O_{y}$ of $X$ such that $K \subset \bigcup_{y \in Y} O_{y}$. By Theorem 3.1, there exist $x_{0} \in K$ and $y_{0} \in Y$ such that $x_{0} \in T\left(y_{0}\right)$ and $y_{0} \in Q\left(x_{0}\right)$ which contradicts the condition (iii). The conclusion must hold.

Remark 4.1. Theorem 4.1 improves and generalises Theorem 3.3 of Mehta and Sessa [14].

Corollary 4.1. Let $\left(X,\left\{\Gamma_{A}\right\}\right)$ be a compact $H$-space and $G: X \rightarrow 2^{X}$ a preference correspondence such that

(i) for each $x \in X, x \notin H-\operatorname{co}(G(x))$

(ii) for each $y \in X, Q^{-1}(y)$ contains a compactly open subset $O_{y}$ of $X$ $\left(O_{y}\right.$ may be empty for some $y$ ) such that $X=\bigcup_{y \in Y} O_{y}$, where $Q(x)=$ $H-c o(G(x))$ for each $x \in X$.

Then $G$ has a maximal element in $X$.

Proof: By letting $X=Y=K$ and $T(x)=\{x\}$ for each $x \in X$ in Theorem 4.1, the conclusion follows from Theorem 4.1. 


\section{REFERENCES}

[1] J.P. Aubin and I. Ekeland, Applied Nonlinear Analysis (John Wiley and Sons, New York, 1984).

[2] C. Bardaro and R. Ceppitelli, 'Some further generalizations of Knaster-KuratowskiMazurkiewicz theorem and minimax inequalities', J. Math. Anal. Appl. 132 (1988), 484-490.

[3] A. Borglin and H. Keiding, 'Existence of equilibrium actions of equilibrium, A note on the 'new' existence theorems', J. Math. Econom. 3 (1976), 313-316.

[4] F.E. Browder, 'Coincidence theorems, manimax theorems, and variational inequalities', Contemp. Math. 26 (1984), 47-80.

[5] X.P. Ding, 'New generalizations of an H-KKM type theorem and their applications', Bull. Austral. Math. Soc. 48 (1993), 451-464.

[6] X.P. Ding and K.K. Tan, 'Matching theorems, fixed point theorems and minimax inequalities without convexity', J. Austral. Math. Soc. Ser. A 49 (1990), 111-128.

[7] X.P. Ding and K.K. Tan, 'Fixed point theorems and equilibria of noncompact generalized games', in Fixed point theory and applications, (K.K. Tan, Editor) (World Sci. Press, Singapore, London, 1992), pp. 80-96.

[8] X.P. Ding and K.K Tan, 'On equilibria of non-compact generalized games', J. Math. Anal. Appl. 177 (1993), 226-238.

[9] Ky Fan, 'Fixed-point and minimax theorems in locally convex topological linear spaces', Proc. Nat. Acad. Sci. USA 38 (1952), 121-126.

[10] Ky Fan, 'A generalization of Tychonoff's fixed point theorem', Math. Ann. 142 (1961), 305-310.

[11] C.W. Ha, 'Extensions of two fixed point theorems of Ky Fan', Math. Z. 190 (1985), 13-16.

[12] C. Horvath, 'Some results on multivalued mappings and inequalities without convexity', in Nonlinear and convex analysis, (B.L. Lin and S. Simons, Editors) (Marcel Dekker, 1987), pp. 99-106.

[13] H. Komiya, 'Coincidence theorems and Saddle point theorem', Proc. Amer. Math. Soc. 96 (1986), 59-602.

[14] G. Mehta and S. Sessa, 'Coincidence theorems and maximal elements in topological vector spaces', Math. Japon. 47 (1992), 839-845.

[15] S. Park, 'Some coincidence theorems on acyclic multifunctions and applications to KKM theory', in Fixed point theory and applications, (K.K. Tan, Editor) (World Sci. Press, Singapore, London, 1992), pp. 248-277.

[16] S. Sessa, 'Some remarks and applications of an extensions of a lemma of Ky Fan', Comment. Math. Univ. Carolin. 29 (1988), 567-575.

[17] N. Shioji, 'A further generalization of Knaster-Kuratowski-Mazurkiewcz theorem', Proc. Amer. Math. Soc. 111 (1991), 187-195.

[18] E. Tarafdar, 'On nonlinear variational inequalities', Proc. Amer. Math. Soc. 67 (1977), 95-98.

[19] E. Tarafdar, 'A fixed point theorem in $H$-space and related results', Bull. Austral. Math. 
Soc. 42 (1990), 133-140.

[20] E. Tarafdar, 'A Fixed point theorems and equilibrium point of an abstract economy', $J$. Math. Econom. 20 (1991), 211-218.

[21] E. Tarafdar, 'Fixed point theorems in $H$-spaces and equilibrium points of abstract economies', J. Austral. Math. Soc. Ser. A 53 (1992), 252-260.

[22] N.C. Yannelis and N.D. Prabhakar, 'Existence of maximal elements and equilibrium in linear topological spaces', J. Math. Econom. 12 (1983), 233-245.

Department of Mathematics

Sichunn Normal University

Chengdu, Sichuan

People's Republic of China 610066
Department of Mathematics

The University of Queensland

Queensland 4072

Australia 